\title{
Abordaje clínico mínimamente invasivo de fluorosis dental en estadios de TF1 a TF5. Revisión sistemática
}

\section{Minimally invasive clinical approach of dental fluorosis in stages of TF1 to TF5. Systematic review}

\author{
J. Covaleda Rodriguez*, A. Torres Peñuela**, M. Sánchez Esparza**, R. Pineda**, V. Silva Borrero**, \\ D. Parra Galvis***, C. Rodríguez Lara****, S. Aguilera-Rojas*****, I. Revelo Mejía******
}

\section{RESUMEN}

Introducción: El manejo clínico de las lesiones dentales ocasionadas por fluorosis dentalserealizamediantediversastécnicasqueresultansermuyagresivasyconpobres resultados estéticos, por estas razones se plantea como objetivo determinar el tratamiento mínimamente invasivo y estético para fluorosis dental en los estadios de 1 a 5 según el índice de Thylstrup and Fejerskov, descritos en laliteratura.

Metodología: Revisión sistemática de 2.299 artículos, procedentes de cuatro bases de datos: PubMed, Embase, Science Direct y EBSCO; la búsqueda se realizó con ocho términos MeSH y tres conectores booleanos para una selección final de 22 artículos en inglés, español y portugués, publicados entre enero de 2009 y diciembre de 2018.

Resultados: El tratamiento más efectivo para lesiones en estadios TF1 y TF2 fue aclaramiento dental con peróxido de carbamida a $15 \%$ o peróxido de hidrógeno a 35\% durante tres sesiones de 15 minutos cada una, reforzado con peróxido de carbamida a 10\%. Para estadios TF3 y TF4 fue microabrasión con ácido clorhídrico al $6 \%$ y carburo de silicio y/o con aclaramiento dental. Para lesioneTF5 fue la técnica combinada de macro y microabrasión con ácido fosfórico a $37 \%$ o ácido clorhídrico a $6 \%, 15 \%$ y $18 \%$, aclaramiento y aplicación de resina infiltrante.

Conclusión: Existen bases científicas que indican que el tratamiento de la Fluorosis es directamente proporcional al estadio de la lesión.

PALABRAS CLAVE: Fluorosis dental, tratamiento dental estético, microabración dental, macroabración dental, composite infiltrativo, blanqueamiento dental, hipoplasia dental y manejo dental.

\section{ABSTRACT}

Introduction: The clinical management of dental lesions caused by dental fluorosis is carried out through various techniques that are very aggressive and with poor aesthetic results. For these reasons, the aim is to determine the minimally invasive and aesthetic treatmentfordentalfluorosisinstagesof 1to5accordingtotheThylstrupandFejerskov index, described in theliterature.

$*$

$* *$

$* * *$

$* * * *$

Odontólogo, Especialista en Rehabilitación oral, Docente posgrado UNICOC.

Residentes de la Especialización en Prostodoncia UNICOC.

Odontóloga, Especialista en Epidemiologia, Docente investigadora UNICOC

Odontólogo, Especialista en Periodoncia y Prostodoncia. Mg. en Educación. Director posgrado

en Prostodoncia UNICOC.

Odontóloga, Especialista en Cirugía Oral, y Mg. En Ciencias Básicas biomédicas.

Docente-Investigador Directora Investigación y Gestión del Conocimiento UNICOC.

*dontóloga, Mg. Administración De Salud, Docente investigadora UNICOC. 
Methodology: Systematic review of 2,299 articles, from four databases: PubMed, Embase, Science Direct and EBSCO; the search was carried out with eight MeSHterms and three Boolean connectors for a final selection of 22 articles in English, Spanish and Portuguese, published between January 2009 and December2018.

Results: The most effective treatment for lesions in stages TF1 and TF2 was dental clearance with carbamide peroxide at $15 \%$ or hydrogen peroxide at $35 \%$ for three sessions of 15 minutes each, reinforced with $10 \%$ carbamide peroxide. For stages TF3 and TF4 it was microabrasion with $6 \%$ hydrochloric acid and silicon carbide and / or with dental clearance. For lesioneTF5 was the combined technique of macro and microabrasion with phosphoric acid at $37 \%$ or hydrochloric acid at $6 \%, 15 \%$ and $18 \%$, clearance and application of infiltrating resin.

Conclusion: There are scientific bases that indicate that the treatment of Fluorosis is directly proportional to the stage of the lesion.

KEY WORDS: Dental fluorosis, Aesthetic dental treatment, Dental microabration, Dental macroabration, Infiltrative composite, Dental bleaching, Dental hypoplasia y Dental management.

Fecha de recepción: 10 de marzo de 2019

Fecha de aceptación: 15 de febrero de 2020

J. Covaleda Rodriguez, A. Torres Peñuela, M. Sánchez Esparza**, R. Pineda, V. Silva Borrero, D. Parra Galvis, C. Rodríguez Lara, S. Aguilera-Rojas, I. Revelo Mejía. Abordaje clínico mínimamente invasivo de fluorosis dental en estadios de TF1 a TF5. Revisión sistemática. 2021; 37, (2): 87-93.

\section{INTRODUCCIÓN}

La fluorosis dental, es un defecto de desarrollo del esmalte ocasionada por el consumo excesivo de fluoruros, por eso se considera una afección endémica en zonas donde el agua tiene concentraciones que superan $1.5 \mathrm{mg} / \mathrm{L} .{ }^{1}$ Este defecto puede suceder a lo largo del desarrollo del diente, tiempo en el que la ingesta de agua, repercute en la formación del esmalte, entre 20 y 36 meses de vida para la dentición permanente y en la vida intrauterina para la dentición temporal, aunque en esta última la fluorosis es menosfrecuente ${ }^{2}$. En el proceso de amelogénesis se requiere que los ameloblastos o células formadoras de esmalte transporten minerales $\left(\mathrm{HPO}^{2}, \mathrm{CO}^{2}, \mathrm{Na}^{+}, \mathrm{F}^{-}\right)$y aminoácidos del plasma al interior de la célula, para originar las proteínas del esmalte y secretarlas a la matriz extra celular y formar los cristales de hidroxiapatita. ${ }^{3}$

Las principales proteínas son la amelogenina, ameloblastina, enamelina y tuftelina; metaloproteínas de matriz como la MMP-20 y calicreína ${ }^{4}$ (KLK4). Las MMP-20 degradan proteínas en la etapa de secreción y maduración, pero al iniciar la maduración dejan de producirse y comienzan a formar KLK4 que modifican la matriz proteica del esmalte, remodelan la zona orgánica para que ese espacio sea ocupado por la parte inorgánica y los cristales de hidroxiapatita tengan mayor grosor. ${ }^{3}$ La incorporación del flúor $\left(\mathrm{F}_{2}\right)$ a la estructura del esmalte, genera alteraciones en el transporte vesicular de los ameloblastos y en la degradación intracelular de proteínas de la matriz, retrasando la eliminación de proteínas, principalmente de amelogeninas, lo que impide el engrosamiento de los cristales y conduce a una mineralización incompleta; además inhibe la calicreina, responsable de la reabsorción de la parte orgánica y por esta razón no se realiza en los tiempos y en las cantidades necesarias, comoresultado la mineralización del esmalte genera una estructura con cristales débiles. ${ }^{4}$ A nivel macroscópico se observa anomalías en la superficie y subsuperfice del esmalte que se caracterizan por incremento en la permeabilidad y la generación de manchas opacas blanquecinas, estriaciones moteadas y fisuras transversales de la superficie del esmalte dental, ${ }^{5}$ así como fosas discontinuas con zonas de sub mineralización, que forman pigmentaciones con el transcurso del tiempo hasta formar manchas de color marrón que pueden ocasionar fracturas de la superficie y alteración de la morfología dental con aparición de otras patologías orales. ${ }^{6}$

En Colombia los dos primeros estudios decenales de salud bucodental realizados en 1965-66 y 197780 , informaron que $97,6 \%$ de los colombianos te- 
nían historia de caries dental por eso se implementó como estrategia preventiva y como único vehículo de administración de fluoruros la sal de consumo humano. Con el tercer Estudio Nacional de Salud Bucal (ENSABIII) de 1998, se identificó una prevalencia de $11,5 \%$ de fluorosis de leve a moderada y según el Índice Comunitario de Fluorosis de Dean (ICF) se considera que un país presenta fluorosis como problema de salud pública, cuando sus valores superan el 0,6\%. Posteriormente el ENSAB IV reportó un ICF de $0,1 \%$ a los 5 años; $0,9 \%$ a los 12 años y $0,84 \%$ a los 15 años. con disminución o aumento de la patología. Los investigadores clínicos y de salud pública después de 70 años de seguimiento, reportaronque $0.5 \mathrm{mg} / \mathrm{L}$ de fluoruro en el agua de consumo son suficientes para prevenir la caries dental y que el exceso en los primeros años de vida incrementa el riesgo de fluorosis dental afectando incluso la salud general. ${ }^{7}$

Hay que partir de la premisa que el fluoruro debe estar en el agua de consumo para que el diagnóstico definitivo sea fluorosis, en vista que los índices han sido desarrollados con el fin de describir sus características clínicas, definir la severidad de la enfermedad, explicar su comportamiento y decidir la terapéutica indicada. El índice Thylstrup y Fejerskov (TF) de 1978, clasifica las lesiones con base en la histopatología de la fluorosis dental, muestra la secuencia lógica de los diferentes estadios para lograr la planificación acertada del tratamiento. Este índice se diferencia de otros porque hace una descripción detallada de las lesiones y la profundidad en el esmalte, medida en micras ya grupadas en 10 estadios que van desde TF0 o esmalte normal, hasta TF9 esmalte con fluorosis severa. Otro mecanismo para clasificar las lesiones ocasionadas por la fluorosis es la transiluminación en lesiones superficiales y profundas según sean mayores o menores de $30 \mathrm{mi}-$ cras. Para la selección del tratamiento adecuado, es necesario tener en cuenta la clasificación de la lesión y su correcto diagnóstico. La fluorosis dental afecta tanto los dientes anteriores como los posteriores, pero el segmento anterior es elmotivo de consulta preferente para recibir un tratamiento estético y funcional. En las últimas décadas la estética en dientes anteriores se ha convertido en una de las consideraciones más importantes en la práctica odontológica, porque ésta condición afecta la calidad de vida y tiene efectos psicosociales en las personas. ${ }^{8,9}$

El manejo de estas lesiones se ha venido realizando mediante tratamientos invasivos, pero el problema es que la mayoría de los pacientes que padecen la fluorosis dental son jóvenes y estos procedimientos resultan en la pérdida injustificada y prematura de los dientes afectados. ${ }^{10,11} \mathrm{~A}$ pesar que estas técnicas han logrado ser efec-

Cuadro 1. Estrategia de búsqueda en bases de datos seleccionadas

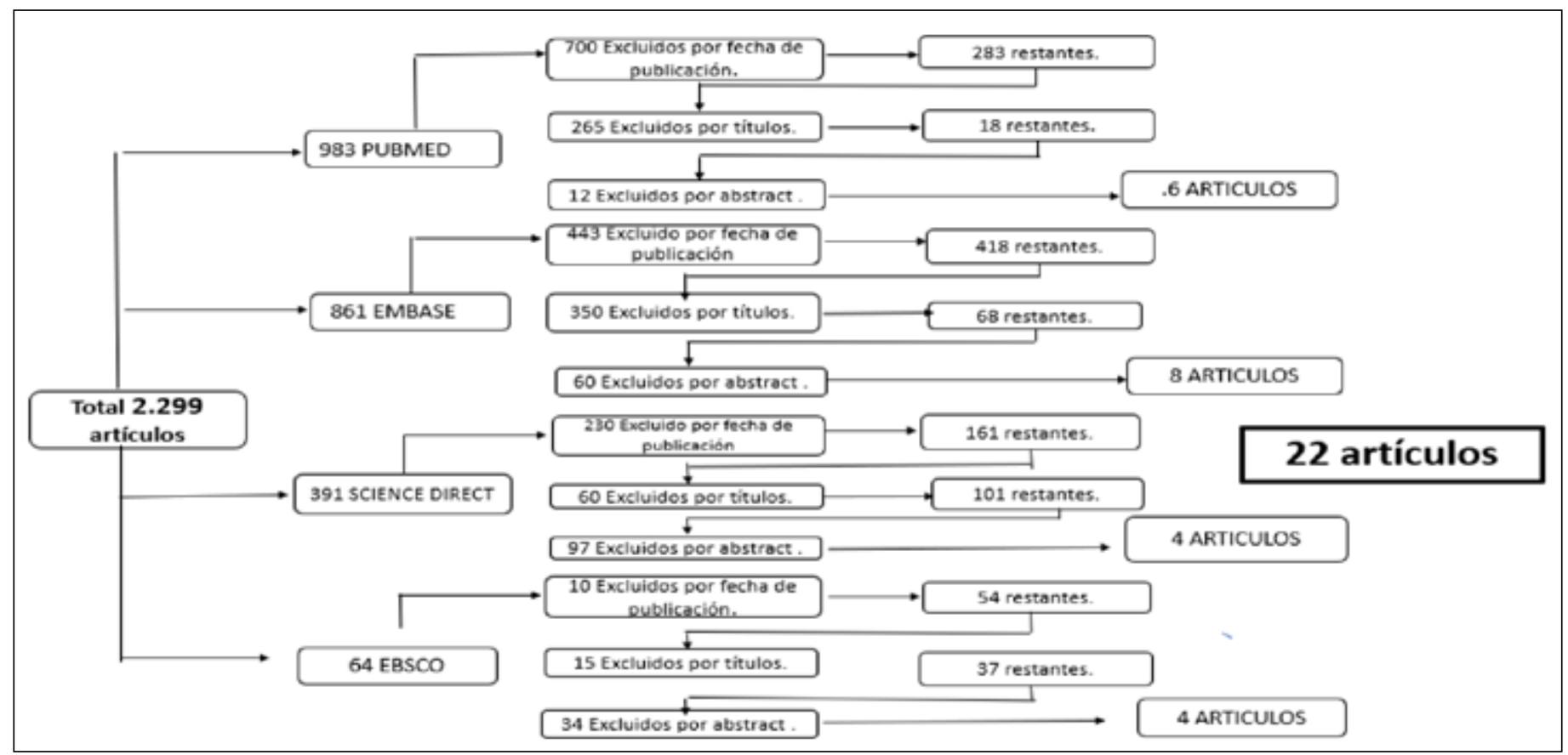


tivas para eliminar algunas pigmentaciones, no se conoce un manejo claro para cada uno de los estadios. En la actualidad se utiliza diversas técnicas para tratar este tipo de lesiones generalmente con procedimientos que provocan micro-abrasión del esmalte, previo al aclaramiento dental y obturaciones con resina. Sin embargo, estas técnicas resultan ser muy agresivas y con pobres resultados estéticos, por estas razones se plantea comoobjetivo de este proyecto identificar las terapéuticas que sean mínimamente invasivas y que aseguren resultados estéticos en los estadios de 1 a 5 de la Fluorosis. ${ }^{12,13}$

\section{METODOLOGIA}

El estudio es una revisión sistemática de 2.299 artículos. Cuatro revisores realizaron de forma independiente la búsqueda sistemática en Pub Med, Embase, Science Direct y bases de datos EBSCO. También se realizó una búsqueda manual adicional, de artículos IN-Press en revistas de alto impacto y artículos no identificados por búsqueda electrónica. (Cuadro 1).

Se utilizó los términos MeSH: Dental fluorosis, Aesthetic dental treatment, Dental microabration, Dental macroabration, Infiltrative composite, Dental bleaching, Dental hypoplasia y Dental management. Una vez definidas las palabras clave, se estableció las estrategias de búsqueda utilizando conectores booleanos AND, OR y NOT con distribución de las palabras en ordenlógico.

Luego de la revisión se seleccionó un total de 22 artículos bajo los siguientes criterios de inclusión: textos científicos cuya población de referencia sea humana, que se encuentren en rango de edad de 6 a 35 años, en idioma inglés, español y portugués, publicados desde el 1 enero de 2009 hasta diciembre de 2018, con diseño de estudio relacionado con metodologías observacionales analíticas (casos y controles - cohortes), ensayos clínicos, reporte de casos y población de referencia con diagnóstico de fluorosis dental en estadios desde TF1 hasta F5. Y los criterios de exclusión fueron: Artículos científicos que refieran obturaciones o fracturas dentales de la superficie vestibular.

Para el análisis se aplicó una lista de chequeo a cada uno de los artículos según el tipo de estudio; Strobe: Estudios observacionales y analíticos.
Care: Reporte de casos clínicos y Consort: Ensayos clínicos aleatorizados. Estas listas se aplicaron para consolidar la validez interna y externa, controlar los sesgos y obtener validez en los resultados. Posteriormente se incorporó los artículos a una base de datos diseñada en el programa Excel versión 2010.

\section{RESULTADOS}

El consenso de los tratamientos reportados es el siguiente:

Microabrasión. Debe realizarse bajo aislamiento absoluto, con aplicación aproximadamente 1 mmde capa de una suspensión micro abrasiva compuesta por ácido clorhídrico al 6\%,15\% y $18 \%$ o ácido fosfórico al $37 \%$ y carburo de silicio o piedrapómez. (Cuadro 2).

\begin{tabular}{|c|c|c|c|}
\hline AÑo & AUTOR & $\mathbf{N}$ & TF \\
\hline 2013 & Maud D. y cols. ${ }^{14}$ & 4 & TF3 \\
\hline 2013 & Mahshid B. y cols. ${ }^{15}$ & 10 & TF3-TF4 \\
\hline 2010 & Nevares-Rascó M. y cols. ${ }^{16}$ & 1 & TF5 \\
\hline 2015 & Natera A, y cols. ${ }^{17}$ & 1 & TF 5 \\
\hline 2010 & Vishal K. y cols. ${ }^{18}$ & 1 & TF3-TF4 \\
\hline 2014 & Castro KS y cols. ${ }^{19}$ & 70 & TF1-TF5 \\
\hline 2013 & Neelam M. y cols. ${ }^{20}$ & 1 & TF3-TF4 \\
\hline 2010 & Howard E. Strassler y cols. ${ }^{21}$ & 1 & TF3-TF4 \\
\hline 2017 & Muniz N. y cols. ${ }^{22}$ & 1 & TF4 \\
\hline 2014 & Xavier L, Batista J, cols. ${ }^{23}$ & 1 & TF4-TF5 \\
\hline 2017 & Gupta A y cols. ${ }^{12}$ & 90 & TF4 \\
\hline 2013 & Celik E. y cols. ${ }^{24}$ & 14 & TF1 -TF5 \\
\hline 2013 & Bassir M. y cols. ${ }^{31}$ & 7 & $\mathrm{TF} 3-\mathrm{TF} 4$ \\
\hline 2010 & Sherwood I y cols ${ }^{25}$ & 3 & TF1- TF5 \\
\hline 2013 & Celik E y cols. ${ }^{26}$ & 10 & TF1-TF5 \\
\hline
\end{tabular}

Aclaramiento dental. Procedimiento que se realiza con Hipoclorito de sodio, peróxido de hidrógeno y peróxido de carbamida en diferentes concentraciones. El peróxido penetra al diente por ósmosis y a través de la permeabilidad actúa directamente sobre las moléculas pigmentadas y promueve la remoción de las estructuras moleculares hidrosolubles más simples generando cambio en el color. ${ }^{14}$ (Cuadro 3).

Resina infiltrante. La resina fotopolimerizable de baja viscosidad permite rápida penetración en el esmalte poroso capaz de inhibir la desmineralización, enmascara las lesiones y oblitera las microporosidades dentro de la lesión. ${ }^{15}$ (Cuadro 4). 
J. Covaleda Rodriguez, A. Torres Peñuela, M. Sánchez Esparza, R. Pineda,

V. Silva Borrero, D. Parra Galvis, C. Rodríguez Lara, S. Aguilera-Rojas, I. Revelo Mejía. Abordaje clínico mínimamente invasivo de fuorosis dental en estadios de TFI a TF5. Revisión sistemática.

Cuadro 3. Tratamiento con aclaramiento dental

\begin{tabular}{|c|c|c|c|c|}
\hline AÑO & AUTOR & $\mathbf{N}$ & TF & TÉCNICA \\
\hline 2017 & Muniz N. y cols. ${ }^{22}$ & 1 & TF4 & Aclaramiento con peróxido de hidrógeno al 35\%. + Peróxido de Carbamida 16\% \\
\hline 2012 & Guedes D. y cols. & 1 & TF5 & Aclaramiento de peróxido de hidrógeno al 35\% \\
\hline 2013 & Tirlet G, cols. ${ }^{27}$ & 4 & TF2 y TF3 & Aclaramiento con peróxido de carbamida al $10 \%$ \\
\hline 2014 & Castro KS. y cols. ${ }^{19}$ & 70 & TF1 a TF7 & Aclaramiento con peróxido de carbamida al $10 \%$ \\
\hline 2017 & Gupta A. y cols. ${ }^{12}$ & 90 & TF1 a TF4 & Aclaramiento con Peróxido de hidrógeno al 35\% activado con luz LED \\
\hline 2017 & Gupta A. y cols. ${ }^{12}$ & 9 & TF1 a TF4 & Aclaramiento con ácido fosfórico al $37 \%$ más hipoclorito de sodio al $5 \%$ \\
\hline 2017 & Gugnani N. y cols. ${ }^{11}$ & 16 & TF1 a TF4 & Aclaramiento con peróxido de hidrogeno al 35\% \\
\hline 2010 & Sherwood I. y cols. ${ }^{25}$ & 3 & TF3 y TF4 & Aclaramiento con una solución de éter anestésico, clorhídrico (36\%) y peróxido de hidrógeno 30\% \\
\hline
\end{tabular}

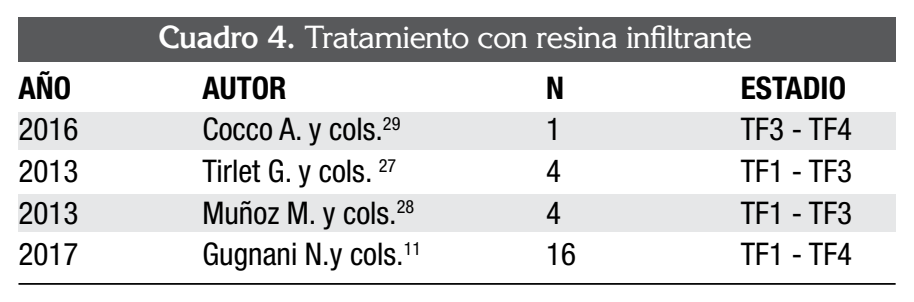

Técnicas combinadas. Macroabrasión en combinación con microabrasión, aclaramiento y resina infiltrante. (Cuadro 5).

*Técnica combinada de macro, microabrasión y aclaramiento. Se realiza primero la microabrasión del esmalte para generar una superficie homogénea, luego aclaramiento.

(19) Después se aplica el flúor neutro por 1 minuto para remineralizar el esmalte y evitar sensibilidad posterior. (Cuadro 6)
Efectividad en la estética de los tratamientos/ estadio. TF1 y TF2 se trataron con aclaramiento dental a base de hipoclorito de sodio, peróxido de hidrogeno al 35\% y peróxido de carbamida al $10 \%$; microabrasión con ácido fosfórico al 37\%; ácido clorhídrico al $6.6 \%$ y resina infiltrante. La efectividad reportada en su mayoría fue alta y los resultados estéticos satisfactorios. (Cuadro 7).

\section{CONCLUSIONES}

En la actualidad se presentan diversos tratamientos para las lesiones de fluorosis dental, en primera instancia es necesario un correcto diagnóstico, determinar el estadio de la lesión y elegir el tratamiento indicado.

El desconocimiento sobre tratamientos mínima-

\begin{tabular}{|c|c|c|c|c|}
\hline AÑO & AUTOR & $\mathbf{N}$ & \multicolumn{2}{|l|}{ TÉCNICA } \\
\hline 2012 & Guedes D. y cols.30 & 1 & \multicolumn{2}{|c|}{ Macroabrasión + microabrasión con Ácido clorhídrico 6\% y aclaramiento con peróxido de hidrógeno 35\%. } \\
\hline 2010 & Howard E. y cols. 21 & 1 & \multicolumn{2}{|c|}{ Macroabrasión + microabrasion con ácido clorhídrico $6.6 \%$, carburo de sílice y peróxidode carbamida 15\% } \\
\hline 2013 & Celik E. y cols.24,26 & 10 & \multicolumn{2}{|c|}{ Macroabrasión y microabrasión con ácido clorhídrico al 6.6\% } \\
\hline 2012 & Muñoz M.y cols.28 & 4 & \multicolumn{2}{|c|}{ Macroabrasión, microabrasion con instrumento rotatorio con ácido clorhídrico al 15\% y resina infiltrante. } \\
\hline \multicolumn{5}{|c|}{ Cuadro 6. Técnica combinada de macro, microabrasión y aclaramiento } \\
\hline 2012 & Guedes D. y cols. ${ }^{30}$ & 1 & TF5 & $\begin{array}{l}\text { Macroabrasión más microabrasión con Ácido clorhídrico al } 6 \% \text { y aclaramientodental con } \\
\text { peróxido de hidrógeno al } 35 \% \text {. }\end{array}$ \\
\hline 2013 & Tirlet G. y cols. ${ }^{27}$ & 4 & TF2 & Aclaramiento con peróxido de carbamida al $10 \%$ más resina infiltrante \\
\hline 2014 & Castro KS. y cols. ${ }^{19}$ & 70 & TF1 TF5 & Microabrasión con ácido fosfórico al 37\% más aclaramiento dental con peróxido de carbamida a 10\% \\
\hline 2012 & Neelam M. y cols. ${ }^{20}$ & 1 & TF4 & Aclaramiento con peróxido de hidrógeno, 35\%, micro abrasión con ácidoclorhídrico 18\% \\
\hline 2010 & Howard E. y cols. ${ }^{21}$ & 1 & TF4 TF5 & $\begin{array}{l}\text { Macroabrasión más microabrasión con ácido clorhídrico al } 6.6 \% \text {, carburo desílice y aclaramiento con } \\
\text { peróxido de carbamida a } 15 \%\end{array}$ \\
\hline 2014 & Muniz N. y cols. ${ }^{22}$ & 1 & TF4 & $\begin{array}{l}\text { Microabrasión con ácido fosfórico al } 37 \% \text { más piedra pómez. Aclaramiento con peróxido de } \\
\text { hidrógeno al } 35 \% \text { y peróxido de carbamida al } 16 \%\end{array}$ \\
\hline 2017 & Gupta A. y cols. ${ }^{12}$ & 90 & TF5 & $\begin{array}{l}\text { Mlcroabrasión Ácido clorhídrico al 15\% + Carburo de silicio más aclaramientocon peróxido } \\
\text { de carbamida al } 44 \% \text {. }\end{array}$ \\
\hline
\end{tabular}




\begin{tabular}{|c|c|c|c|c|}
\hline ESTADIO & TRATAMIENTO & EFECTIVIDAD & AUTORES & AÑO \\
\hline TF1 & Aclaramiento & Alta & Gupta A. y cols.12 & 2017 \\
\hline TF1 & Aclaramiento & Alta & Gugnani N. y cols.11 & 2013 \\
\hline TF1 & Aclaramiento & Alta & Tirlet G. y cols.27 & 2013 \\
\hline TF1 & Microabración & Alta & Celik E. y cols.24, 26 & 2013 \\
\hline TF1 & Microabración & Media & Castro K. y cols.19 & 2014 \\
\hline TF2 & Aclaramiento & Baja & Gupta A. y cols.12 & 2017 \\
\hline TF2 & Aclaramiento & Alta & Gugnani N. y cols.11 & 2017 \\
\hline TF2 & Resina Infiltrante & Alta & Tirlet G. y cols.27 & 2013 \\
\hline TF2 & Resina Infiltrante & Alta & Muñoz M. y cols.28 & 2017 \\
\hline TF2 & Microabración & Alta & Celik E. Y cols.24,26 & 2013 \\
\hline TF3 & Microabración & Media & Castro KS. y cols.19 & 2014 \\
\hline TF3 & Microabración & Media & Vishal B. y cols.18 & 2013 \\
\hline TF3 & Microabración & Alta & Celik E. y cols.24,26 & 2013 \\
\hline TF3 & Microabración & Baja & Mahsid B. y cols.15 & 2013 \\
\hline TF3 & Microabración & Baja & Bassier M., y cols.32 & 2013 \\
\hline TF3 & Aclaramiento & Alta & Gupta A. y cols.12 & 2017 \\
\hline TF3 & Aclaramiento & Alta & Gugnani N. y cols.11 & 2017 \\
\hline TF3 & Aclaramiento & Alta & Tirlet G. y cols..27 & 2013 \\
\hline TF3 & Resina Infiltrante & Alta & Tirlet G. y cols.27 & 2013 \\
\hline TF3 & Técnica Combinada & Alta & Gugnani N. y cols.11 & 2017 \\
\hline TF3 & Técnica Combinada & Alta & Sherwood I. y cols.25 & 2010 \\
\hline TF3 & Técnica Combinada & Alta & Neelam M. y cols.20 & 2012 \\
\hline TF3 & Técnica Combinada & Alta & Castro K. y cols.19 & 2014 \\
\hline TF3 & Técnica Combinada & Alta & Mahud D. y cols.14 & 2013 \\
\hline TF4 & Resina Infiltrante & Baja & Cocco A. y cols. 29 & 2016 \\
\hline TF4 & Resina Infiltrante & Alta & Muñoz M. y cols.28 & 2012 \\
\hline TF4 & Aclaramiento & Alta & Muniz N. y cols.22 & 2017 \\
\hline TF4 & Técnica Combinada & Alta & Gugnani N. y cols.11 & 2017 \\
\hline TF4 & Técnica Combinada & Alta & Sherwood I. y cols.25 & 2010 \\
\hline TF4 & Microabración & Alta & Xavier L Baptista y cols.23 & 2014 \\
\hline TF4 & Microabración & Baja & Celik E. Y cols.24,26 & 2013 \\
\hline TF4 & Microabración & Alta & Bassir E. y cols.31 & 2013 \\
\hline TF4 & Microabración & Alta & Mashid B, y cols.15 & 2013 \\
\hline TF4 & Microabración & Alta & Vishal K. y cols.18 & 2013 \\
\hline TF4 & Técnica Combinada & Alta & Howard E., y cols.21 & 2010 \\
\hline TF4 & Técnica Combinada & Alta & Castro KS. y cols.19 & 2014 \\
\hline TF4 & técnica Combinada & Alta & Gupta A. y cols.12 & 2017 \\
\hline TF4 & Aclaramiento & Baja & Neelam M. y cols.20 & 2012 \\
\hline TF5 & Aclaramiento & Baja & Guedes D. y cols.30 & 2012 \\
\hline TF5 & Microabración & Baja & Narvaez M. y cols.16 & 2010 \\
\hline TF5 & Microabración & Media & Xavier L.Baptista y cols.23 & 2014 \\
\hline TF5 & Microabración & Baja & Celik E. y cols.26 & 2013 \\
\hline TF5 & Microabración & Baja & Natera A. y cols.17 & 2015 \\
\hline TF5 & Técnica Combinada & Alta & Castro KS. y cols.19 & 2014 \\
\hline TF5 & Técnica Combinada & Alta & Howard E., y cols.21 & 2010 \\
\hline TF5 & Técnica Combinada & Alta & Guedes D. y cols.30 & 2012 \\
\hline TF5 & Técnica Combinada & Alta & Sherwood I. y cols. 25 & 2010 \\
\hline
\end{tabular}

destrucción del diente a edades tempranas.

La resina infiltrante es una nueva técnica citada en la literatura que aporta resultados en estéticos de las lesiones fluoróticas.

El manejo indicado para lesiones TF1 y TF2 es aclaramiento dental o resina infiltrante; para TF3 y TF4 microabrasión y/o aclaramiento y en TF5 técnica combinada con macro, microabrasión y aclaramiento dental.

\section{REFERENCIAS BIBLIOGRÁFICAS}

1. Ministerio de Salud y Protección Social. Documento técnico perspectiva del uso del flúor vs caries y fluorosis dental en Colombia. Grupo de Enfermedades no Transmisibles Dirección de Vigilancia y Análisis del Riesgo en Salud Pública Instituto Nacional de Salud Versión 3. Bogotá D.C., Colombia, febrero de 2016. Disponible en: https://www.minsalud.gov.co/ sites/rid/Lists/BibliotecaDigital /RIDE/VS/PP/ENT/ perspectiva-uso-fluor.pdf

2. Azpeitia-Valadez M, Rodríguez-Frausto M, y Sánchez-Hernández M. Prevalencia de fluorosis dental en escolares de 6 a 15 años. Rev Med Inst Mex Seguro Soc. 2008; 46 (1): 67-72.

3. Castiblanco GA, Martignon S, Castellanos JE, Mejía WA. Pathogenesis of dental fluorosis: biochemical and cellular mechanisms [Patogénesis de la fluorosis dental: mecanismos bioquímicos y celulares]. Rev Fac Odontol Univ Antioq 2017; 28(2): 408-21.

4. Rivas Gutiérrez Jesús, Huerta Vega Leticia. Fluorosis dental: Metabolismo, distribución y absorción del fluoruro. Revista ADM, noviembre - diciembre de 2005 Vol. LXII, (6)225-29.

5. Sukhabogi J R, Parthasarathi P, Anjum S, Shekar B, Padma C M, Rani A S. Dental fluorosis and dental caries prevalence among 12 and 15-year-old school children in Nalgonda District, Andhra Pradesh, India. Ann Med Health Sci Res. 2014 Sep-Oct; 4(Suppl 3): S245-52.

6. Agudelo-Suárez AA, Martínez-Flórez LM, Madrid-Gutiérrez LM, Vivares-Builes AM, Rocha-Buelvas A. Panorama de la fluorosis dental en Colombia: una revisión exploratoria de la literatura. Univ Odontol. 2013 Ene-Jun; 32(68): 133-45.

7. Ministerio de Salud y Protección Social. IV Estudio Nacional de Salud Bucal ENSAB - IV 20132014. Bogotá. Ministerio de Salud y Protección Social;2015. Disponible en: https://www.minsalud. gov.co/sites/rid/Lists/BibliotecaDigital/RIDE/VS/PP/ ENSAB-IV-Situacion-Bucal-Actual.pdf[ Links ]

8. Espinosa Fernández Roberto., Valencia Hitte Roberto, Israel Ceja Andrade. Fluorosis dental. Etiología, diagnóstico y tratamiento. Madrid, España. Ripano Editorial Médica. 2012. Libro impreso: español (spa): 1a. edición.

9. Howard E. Strassler; Mark L. Pitel.UsingFiber-OpticTransilluminationasadiagnosticaidindentalpractice. Compen- mente invasivos, conlleva a realizar procedimientos restaurativos con carillas o coronas generando mayor desgaste del esmalte dental, que acelera la 
J. Covaleda Rodriguez, A. Torres Peñuela, M. Sánchez Esparza, R. Pineda,

V. Silva Borrero, D. Parra Galvis, C. Rodríguez Lara, S. Aguilera-Rojas, I. Revelo Mejía. Abordaje clínico mínimamente invasivo de fluorosis dental en estadios de TF1 a TF5. Revisión sistemática.

dium, february 2014. Volume 35, Issue 2.

10. Chávez Pérez Rita. Tratamiento con ácido clorhídrico en pacientes con fluorosis dental. Revista ADM2014; 71(4):202-06. Disponible en:https://www.medigraphic. com/pdfs/adm/od-2014/od144j.pdf

11. Gugnani N, Pandit IK, Gupta M, Gugnani S, Soni S, Goyal V. Comparative evaluation of esthetic changes in nonpitted fluorosis stains when treated with resin infiltration, in-office bleaching, and combination therapiesJ Esthet Restor Dent. 2017 Sep;29(5):317-24. doi: 10.1111/ jerd.12312. Epub 2017 Jun 27.

12. Aarushi Gupta, Renuka Dhingra, Payal Chaudhuri, Anil Gupta. Acomparisonofvariousminimallyinvasivetechniquesfortheremovalofdental fluorosis stains in children. Journal of Indian society of pedodontics and preventive dentistry. 2017. Volume:35. (Issue:3): 260-68.

13. Browne Deirdre, Whelton Helen, O'Mullane Denis. Fluoride metabolism and fluorosis. Journal of Dentistry. 2005; (33):177-86.

14. Maud Denis, Anthony Atlan, Elsa Vennat, Gil Tirlet, Jean-Pierre Attal. White defects on enamel: Diagnosis and anatomopathology: Two essential factors for proper treatment (part 1). International Orthodontics. 2013, 11 (2): $139-65$.

15. Mahshid Mohammadi Bassir and Golnaz Bagheri. Comparison between phosphoric acid and hydrochloric acid in microabrasion techniqueforthetreatmentofdentalfluorosis.J Conserv Dent. 2013 Jan-Feb; 16(1): 41-4. doi: 10.4103/0972-0707.105297

16. Nevárez-Rascón Martina, Julio Villegas-Ham, Nelly Molina-Frechero, Enrique Castañeda-Castaneira, Ronell Bologna-Molina, Alfredo Nevárez-Rascóny. Tratamiento para manchas por fluorosis dental por medio de microabrasión sin instrumentos rotatorios, Rev. CESOdont. 2010; 23(2) 61-6.

17. Natera Alfredo, Da Silva Andreina, Fernández Mariana, Montilla María A, Moukel Jennifer, Rodríguez Luz, Vivas Sara. Tratamiento de fluorosis con microabrasión del esmalte. Reporte de un caso clínico índice TF5. Odous Científica. ISSN:1315 2823, Julio-diciembre 2015.16(2):51-7.

18. Vishal Khandelwal, Ullal Anand Nayak, Prathibha Anand Nayak, and Nupur Ninawe. Aesthetic management of dental fluorosis. Brithis medical journal. Published online. 2013 May 22. doi: 10.1136/bcr-2013-010029.

19. Castro KS, Ferreira AC, Duarte RM, Sampaio FC, Meireles SS. Acceptability, efficacy and safety of two treatment protocols for dental fluorosis: A randomized clinical trial. Journal of dentistry. Jan 2014, 42(8):938-44.

20. Neelam Mittala, Jyoti Jain. A conservative approach for management of fluorosed anterior teeth. Indian Journal of Dentistry, 2012 April -June 3 (2): 118-21.
21. Howard E. Strassler. Clinical case report: Treatment of mild-to- moderate fluorosis with a minimally invasive treatment plan, functional esthetics y restorative dentistry. Compendium Jan/Feb 2010, Vol. 31, Issue 1.

22. Muniz N. y cols. Técnicas conservadoras para tratamiento de fluorosis dentaria: Relato de caso clínico. Dental Press Publish Shing-J Clin Dent. Oct2017 14(4): 48-57.

23. Xavier de Oliveira Luísa Mara, João Batista Novaes-Júnior, Ivan Doche Barreiros, Saul Martins Paiva, Carolina Castro Martins. Tratameto de fluorose dentaria moderada com a técnica de microabrasao de esmalte com ácido cloridrico $6 \%$ e carbeto de silicio: relato de caso clinico. Arq Odontol, Bello Horizonte. 2014. 50 (3):142-48.

24. Celik EU, Yildiz G, Yazkan B.Clinical evaluation of enamel microabrasion for the aesthetic managennent of Mild-to-Severe Dental Fluorosis. J Esthet Restor Dent. 2013, Dec. 25(6):422-32,2013. doi: 10.1111/ jerd.12052. Epub 2013 Sep 5.

25. Sherwood I Anand. Fluorosis varied treatment options. J Conserv Dent 2010, Jan-Mar; 13(1): 47-53. doi: 10.4103/0972-0707.62631

26. Celik EU, Yıldız G, and Yazkan B. Comparison of Enamel Microabrasion with a Combined Approach to the Esthetic Management of Fluorosed Teeth. Operative Dentistry: September/October 2013, Vol. 38 (5): E134-43. https:// doi.org/10.2341/12-317-C

27. Tirlet G, Chabouis HF, Attal JP Infiltration, a new therapy for masking enamel white spots: a 19-month follow- up case serie. Eur J Esthet Dent. 2013 Summer;8(2):180-90.

28. Muñoz MA, Arana-Gordillo LA, Gomes GM, Gomes OM, Bombarda NH, Reis A, Loguercio AD.Alternative Esthetic Management of Fluorosis and Hypoplasia Stains: Blending Effect Obtained with Resin Infiltration Techniques. J Esthet Restor Dent. 2013 Feb;25(1):32-9. doi: 10.1111/ j.1708-8240.2012.00527. x. Epub 2012 Jul 10.

29. Cocco A, Lund RG, Torre E, Martos J. Treatment of Fluorosis Spots Using a Resin Infiltration Technique: 14-month Follow-up.Oper Dent. 2016 Jul-Aug;41(4):357-62. doi: 10.2341/14-335-S.

30. Gueedes D, Michele Ketlen. Re-establishing Esthetics of Fluorosis-Stained Teeth Using Enamel Microabrasion and Dental Bleaching Techniques, The EuropeanJournal Esthetic Dentistry. 2012, volume 7, (2):130-37

31. Bassir M, Bagheri G. Comparison between phosphoric acid and hydrochloric acid in microabrasion technique for the treatment of dental fluorosis. J Conserv Dent 201, $(16): 41-3$

\section{DIRECCIÓN DE CONTACTO:}

iarevelom@unicoc.edu.co Teléfono celular: 3006574862. 